\title{
Accurately evaluating Young's modulus of polymers through nanoindentations: A phenomenological correction factor to the Oliver and Pharr procedure
}

Davide Tranchida, Stefano Piccarolo, Joachim Loos, and Alexander Alexeev

Citation: Appl. Phys. Lett. 89, 171905 (2006); doi: 10.1063/1.2364863

View online: https://doi.org/10.1063/1.2364863

View Table of Contents: http://aip.scitation.org/toc/apl/89/17

Published by the American Institute of Physics

\section{Articles you may be interested in}

Elastic modulus of polystyrene film from near surface to bulk measured by nanoindentation using atomic force microscopy

Applied Physics Letters 89, 031925 (2006); 10.1063/1.2234648

Analysis of nanoindentation creep for polymeric materials

Journal of Applied Physics 95, 3655 (2004); 10.1063/1.1651341

Relationships between hardness, elastic modulus, and the work of indentation

Applied Physics Letters 73, 614 (1998); 10.1063/1.121873

Scaling approach to conical indentation in elastic-plastic solids with work hardening Journal of Applied Physics 84, 1284 (1998); 10.1063/1.368196

Finite-element modeling of nanoindentation

Journal of Applied Physics 85, 1460 (1999); 10.1063/1.369178

Nanoindentation hardness measurements using atomic force microscopy

Applied Physics Letters 64, 1653 (1994); 10.1063/1.111949

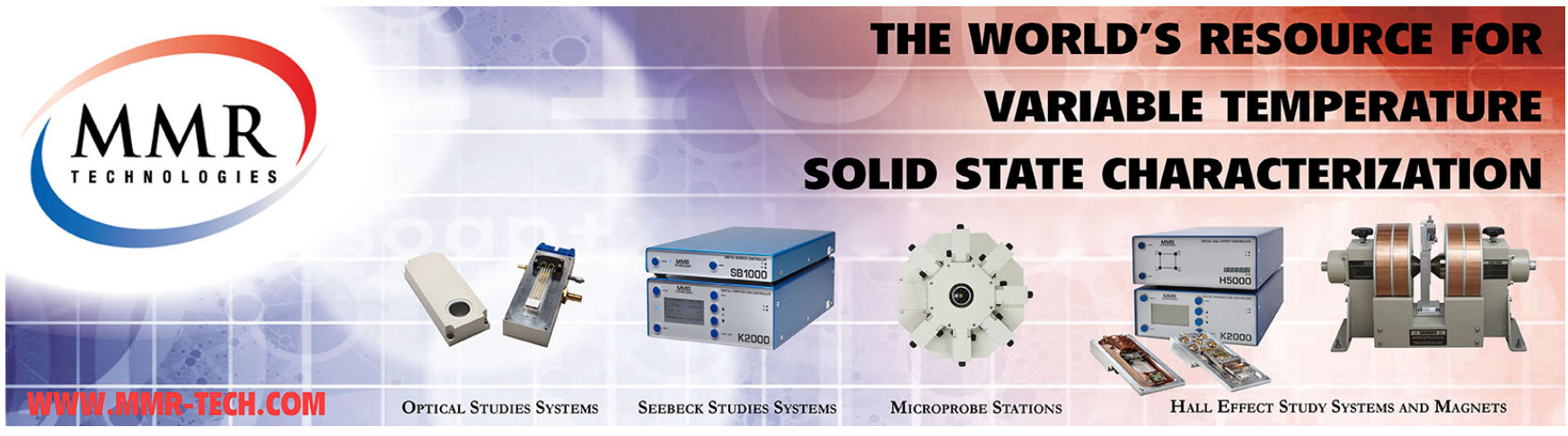




\title{
Accurately evaluating Young's modulus of polymers through nanoindentations: A phenomenological correction factor to the Oliver and Pharr procedure
}

\author{
Davide Tranchida \\ Dipartimento di Ingegneria Chimica dei Processi e dei Materiali, University of Palermo, Viale delle \\ Scienze, 90128 Palermo, Italy and INSTM Udr Palermo, Viale delle Scienze, 90128 Palermo, Italy \\ Stefano Piccarolo ${ }^{\text {a) }}$ \\ Dipartimento di Ingegneria Chimica dei Processi e dei Materiali, University of Palermo, Viale delle \\ Scienze, 90128 Palermo, Italy and INSTM Udr Palermo, Viale delle Scienze, 90128 Palermo, Italy \\ Joachim Loos \\ Department of Chemical Engineering and Chemistry, Eindhoven University of Technology, P.O. Box 513, \\ 5600 MB Eindhoven, The Netherlands and Dutch Polymer Institute, P.O. Box 902, \\ 5600 AX Eindhoven, The Netherlands

\begin{abstract}
Alexander Alexeev
Department of Chemical Engineering and Chemistry, Eindhoven University of Technology, P.O. Box 513, 5600 MB Eindhoven, The Netherlands and NT-MDT, 124460 Moscow, Russian Federation
\end{abstract}

(Received 28 July 2006; accepted 13 September 2006; published online 24 October 2006)

\begin{abstract}
The Oliver and Pharr [J. Mater. Res. 7, 1564 (1992)] procedure is a widely used tool to analyze nanoindentation force curves obtained on metals or ceramics. Its application to polymers is, however, difficult, as Young's moduli are commonly overestimated mainly because of viscoelastic effects and pileup. However, polymers spanning a large range of morphologies have been used in this work to introduce a phenomenological correction factor. It depends on indenter geometry: sets of calibration indentations have to be performed on some polymers with known elastic moduli to characterize each indenter. (C) 2006 American Institute of Physics. [DOI: 10.1063/1.2364863]
\end{abstract}

The standard Oliver and Pharr $(\mathrm{OP})$ procedure $^{1}$ is often used to analyze the unloading part of force curves, i.e., the relation between applied load and penetration depth obtained by a nanoindentation. This procedure has its roots in Sneddon's contact mechanics model ${ }^{2}$ that holds for classical theory of elasticity. However, the capability of polymers to deform lies in their ability to adjust their chain conformation on a molecular level ${ }^{3}$ and the mobility of the macromolecules with their structural arrangements (depending on molecular and morphological constraints) gives rise to a wellknown pronounced time dependence of mechanical properties. Viscoelasticity is therefore intrinsic to the nature of polymers and its effects cannot be eliminated: it has to be properly taken into account with special contact mechanics models and nanoindentation procedures. ${ }^{4}$ As a consequence, the OP procedure can be used to study polymers with a qualitative analysis, comparing different responses to nanoindentation, but quantitative analysis is still a challenging issue.,

Several adjustments have been proposed in the literature, e.g., the calibration of the area function on polycarbonate, but despite these efforts, the OP procedure still usually overestimates Young's moduli of polymers. ${ }^{6}$ The use of high loading rates ${ }^{7}$ and holding times, thus maintaining the maximum load constant for a certain amount of time, can minimize viscoelastic contributions during the unloading curve; nevertheless Young's modulus estimation is not satisfactory. ${ }^{6}$ For this reason, we try in this work to introduce a purely phenomenological correction factor, being aware that it does not allow us to analyze the force curve in a viscoelastic

\footnotetext{
${ }^{a)}$ Electronic mail: piccarolo@unipa.it
}

framework. However, the accuracy of Young's modulus estimation is considerably improved. In summary, seven different polymeric samples characterized by semicrystalline, amorphous, and mesomorphic morphologies were used.

The nanoindentation system used in this study was an assembly of a NT-MDT (Moscow, Russian Federation) atomic force microscope with the standard head replaced by a triboscope indentor system (Hysitron Inc., Minneapolis, $\mathrm{MN}$ ) which can apply a programmed load history on the indenter.

A standard fused silica sample was used to calibrate instrument compliance as well as the area function of a Berkovich indenter according to OP. ${ }^{1}$ Although spherical indenters are often employed in the literature in order to induce small penetration depths in polymers, we chose in this work a Berkovich indenter to comply with the standard OP procedure, provided that the applied loads were tiny enough to obtain penetration depth smaller than $200 \mathrm{~nm}$. Indentations were performed in load-controlled mode after collecting images of the area to be indented in order to check surface roughness in the selected area. Load was initially ramped at constant rate up to the selected value, kept constant for $10 \mathrm{~s}$ and brought back to zero with the same rate. Load varied in the range of $10-140 \mu \mathrm{N}$, and it was applied with high loading rate, 30 or $100 \mu \mathrm{N} / \mathrm{s}$. Thermal drift was measured and corrected for each indentation.

The polymers used in this work covered a broad range of morphologies, glassy amorphous, and semicrystalline ones. Atactic polystyrene (PS), N5000, was kindly supplied from Nova Chemicals, and polycarbonate, Lexan 121R, from GE Plastics. The isotactic polypropylene (iPP), trade name 
T30G, was kindly supplied by Montell. In case of iPP, the samples were prepared by melt solidification under different conditions using cooling rates $(11.5,80,360$, and $888 \mathrm{~K} / \mathrm{s})$ typical of processing. ${ }^{8}$ Under these conditions they develop a broad range of morphologies from purely $\alpha$ monoclinic up to completely mesomorphic at low and high cooling rates, respectively. ${ }^{8}$ Depending on the morphology, whose homogeneity is preserved by the solidification procedure adopted, the samples have different mechanical properties. Young's moduli vary between $1.2 \mathrm{GPa}$ for the semicrystalline one (cooling rate of $11.5 \mathrm{~K} / \mathrm{s}$ ) and $700 \mathrm{MPa}(888 \mathrm{~K} / \mathrm{s}$ ) when the completely mesomorphic morphology is obtained. ${ }^{9}$

Nanoindentation experiments can qualitatively capture the mechanical behavior of different polymers and morphologies. For example, nanoindentations performed at peak load of $30 \mu \mathrm{N}$, not shown here, gives rise to penetration depths of approximately 60, 95, and $110 \mathrm{~nm}$ for amorphous PS, semicrystalline and mesomorphic iPP, respectively. These penetration depths correctly reflect the stiffness of the different morphologies. In order to switch to a quantitative analysis of the force curves, the OP procedure has been used in this work. The contact stiffness $S$, i.e., the slope of the unloading curve, is measured from each force curve; it allows to estimate the reduced modulus $E_{r}$ through the equations

$$
\begin{aligned}
& h_{c}=h_{\max }-\varepsilon P_{\max } / S, \\
& S=\beta \gamma \frac{2}{\sqrt{\pi}} E_{r} \sqrt{A_{c}},
\end{aligned}
$$

where $A_{c}$ is the contact area, $h_{c}$ is the evaluated contact depth, and $h_{\max }$ and $P_{\max }$ are the maximum penetration depth and applied load, respectively. Three correction factors are used in these equations (usually called $\beta$, $\varepsilon$, and $\gamma$ ) and the choice of their values might introduce some difficulties. ${ }^{1,10}$

Recently Troyon and Martin ${ }^{11}$ showed that the value for $\varepsilon$, a geometrical parameter, ${ }^{1,10}$ has to be evaluated for each force curve from the unloading exponent, $m$ using a power fitting law. Even though $\varepsilon$ changes slightly in the normal operating conditions adopted to test metals or ceramics, ${ }^{11}$ where $m$ is bounded between 1 and 2, its variation is definitely debatable for polymers. ${ }^{5,6}$ Therefore, we used the common value of $0.75{ }^{1}$ The correction factor $\beta$, equal to 1.034 (Ref. 12) for the Berkovich indenter, is a purely geometric factor, ${ }^{1,10}$ which takes into account that the indenter is not a perfect cone. The correction factor $\gamma$ arises from the improper account, in Sneddon's solution, for radial material displacement into the contact region. ${ }^{13}$ Following Hay et $a l .,{ }^{13}$ the factor $\gamma$ depends, for a cone indenter, on sample Poisson ratio and the indenter half-included angle. This correction factor takes the value of 1.067 in the case of typical Poisson ratio of 0.3 and the cone with half-included angle such as to give rise to a ratio of displaced volume and penetration depth equivalent to the Berkovich indenter. ${ }^{13}$

The OP analysis was performed with these correction factors and the values for contact stiffness, i.e., the slope of the unloading curve and contact depth from the force curves collected on all the materials studied in this work. Young's moduli obtained from nanoindentations performed in loadcontrolled mode with a loading rate of $30 \mu \mathrm{N} / s$ are plotted in Fig. 1 as the filled series in a 1:1 plot against the elastic moduli measured through macroscopic tensile tests. Even

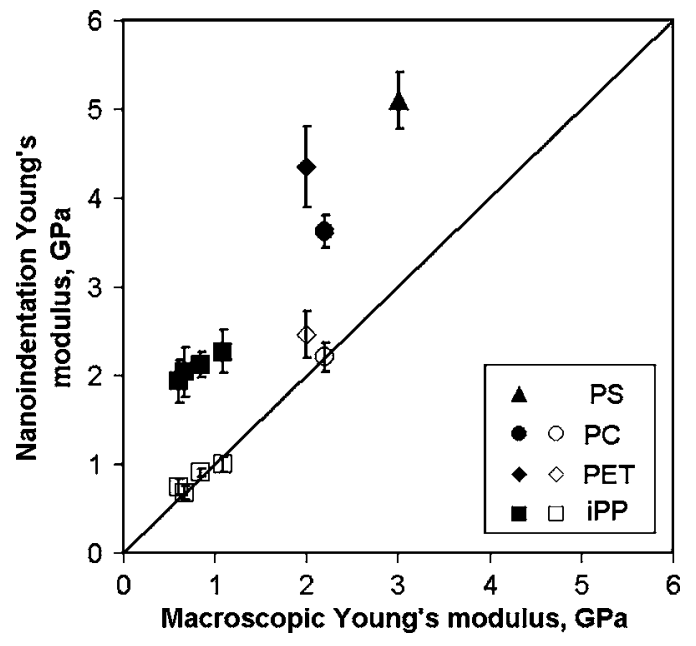

FIG. 1. Comparison between Young's moduli obtained by macroscopic tests and by the Oliver and Pharr procedure, with (empty series) and without (filled series) the $\chi$ correction factor.

tough some difference might be expected since bulk modulus is compared in Fig. 1 to a local measurement, it can be clearly observed that the disagreement is evident, with Young's modulus overestimated up to 3.3 times. $^{6}$ Neither a depressed glass transition at the surface, due to increased mobility, should play a role implying a lower modulus than the bulk.

It is worth mentioning that the magnitude of this deviation is so large that it cannot be due to a wrong choice of the abovementioned correction factors, which can change the results of up to approximately $\pm 15 \%$.

The unusual high values found for the elastic moduli could be explained with the observation that a compression modulus is being measured through nanoindentations, and it can be larger than the one macroscopically measured through tensile tests. However, the magnitude of the deviation (the $E$ from nanoindentation is often several times bigger than the one measured in macroscopic tests) makes this conjecture quite unreasonable when considering that for most polymers compressive and tensile elastic moduli differ at most by $20 \%{ }^{14}$

Once the failure of the OP procedure has been identified, a phenomenological correction factor $\chi$ can be introduced for each force curve, as the ratio of the modulus obtained by the nanoindentation and the expected one,

$$
\chi=E_{\mathrm{OP}} / E_{\text {expected }},
$$

where, in this case, the expected modulus $E_{\text {expected }}$ has been replaced by the macroscopic one measured through tensile tests.

Here we assume that the correction factor depends on the material, i.e., on elastic modulus, and on penetration depth. Since Fig. 1 shows that the deviation from the correct elastic modulus is larger for more compliant samples, an inverse relation can be assumed for $\chi$ on $E$. Concerning penetration depth, a more complex relation is expected due to the nonideality, i.e., bluntness, of the tip which is assumed to be ideally sharp. Identifying the relationship $\chi=\chi(p, E)$ is the aim of the following discussion.

The correction factor $\chi$ is plotted in Fig. 2 against the elastic modulus of the different polymers and fused silica at various penetration depths, i.e., $20,50,100$, and $140 \mathrm{~nm}$. It is 


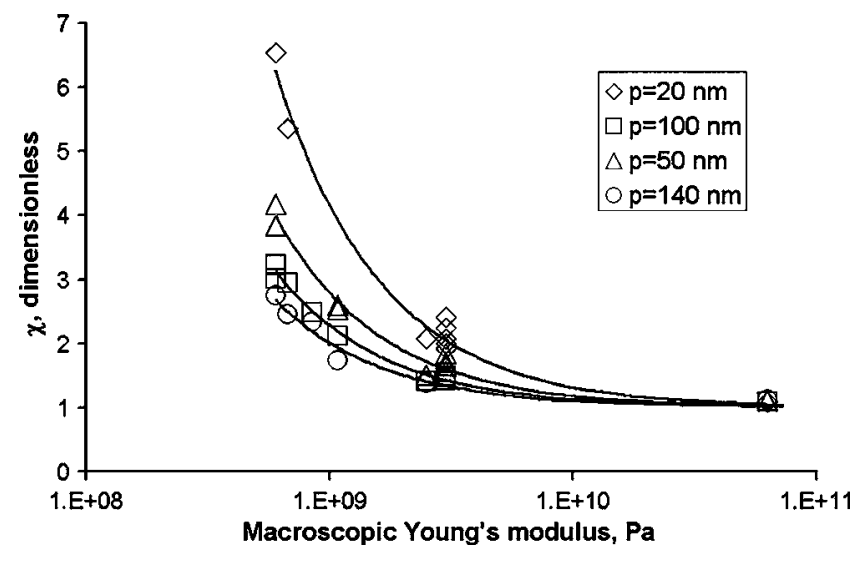

FIG. 2. Relation between the correction factor $\chi$ and the macroscopic Young's modulus at different penetration depths. Fitting lines as in Eq. (3).

worth noting that the very low roughness of the samples, in the order of $1 \mathrm{~nm}$ on a $2 \times 2 \mu \mathrm{m}^{2}$ area, allowed us to use such shallow penetration depths without accounting for contact between single asperities. ${ }^{15}$ The fitting equation to this plot can be

$$
\chi=1+B(p) / E,
$$

where $B$ is the only fitting parameter and depends on penetration depth. The form of Eq. (3) is particularly attractive because $\chi$ becomes 1 for a stiff material such as fused silica, thus not introducing any correction to the OP procedure, while it increases the more compliant is the material. The parameter $B$ dependence on penetration depth was afterwards fitted with a power law relation, as shown in Fig. 3 .

The final dependence for the correction factor is then

$$
\chi=1+a p^{b} / E \text {. }
$$

Although it might seem that the knowledge of the real elastic modulus is needed to evaluate the correction factor $\chi$, a few iterations on Eqs. (2) and (4) lead to the correct elastic modulus once $a$ and $b$ are known through a preliminary calibration.

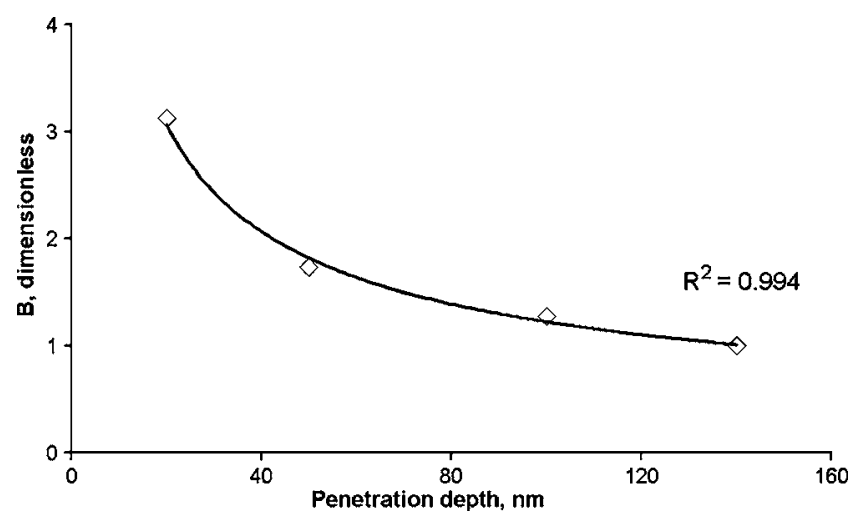

FIG. 3. Power law fitting of the parameter $B$, as obtained from Fig. 2 and Eq. (3), vs penetration depth.
The method was finally checked by calibrating the parameters $a$ and $b$ on the polymer with highest modulus, i.e., $\mathrm{PS}$, in the range of penetration depths of $20-120 \mathrm{~nm}$. The parameters $a$ and $b$ were determined to be $1.134 \times 10^{10}$ and -0.485 , respectively.

Using the correction factor $\chi$, the elastic moduli of all the different polymers studied in this work were recalculated from the data corresponding to the filled series in Fig. 1, leading to the results shown in Fig. 1 as the empty series.

In summary, a satisfactory evaluation of elastic moduli of polymers can be obtained by coupling the common calibrations of the Oliver and Pharr procedure on fused silica with the calibration of the correction factor $\chi$, in the range of penetration depths of interest, on polymer samples of known elastic moduli. It is worth mentioning that particular care must be taken concerning the choice of Young's modulus to be inserted in Eq. (4). Indeed, mechanical properties of polymers are very often assumed on the basis of data in the literature, which can result in large mistakes due to the different molecular characteristics (molecular weight, molecular weight distribution, etc.) as well as, and in particular, to the morphology developed during sample preparation. ${ }^{16}$ For example, avoiding the use of injection moulded semicrystalline samples is highly recommended because thermal gradients, pressure, and shear at the near surface can give rise to a morphology gradient and thus to an inhomogeneous, unknown a priori, structure. ${ }^{17}$

This work was partially funded by the European Science Foundation through a Short Term Scientific Mission in the framework of COST P12, Structuring of Polymers, Action. The authors acknowledge the financial support of the Ph.D. grant of DT by the University of Palermo and the financial support from the Italian Ministry of University and Scientific Research (MIUR, Italy), through the PRIN04 grant.

${ }^{1}$ W. C. Oliver and G. M. Pharr, J. Mater. Res. 7, 1564 (1992).

${ }^{2}$ I. N. Sneddon, Int. J. Eng. Sci. 3, 47 (1965).

${ }^{3}$ V. N. Pokrovskii, Adv. Polym. Sci. 54, 143 (2001).

${ }^{4}$ Y.-T. Cheng and C.-M. Cheng, J. Mater. Res. 20, 1046 (2005).

${ }^{5}$ D. Tranchida and S. Piccarolo, Macromol. Rapid Commun. 26, 1800 (2005).

${ }^{6}$ D. Tranchida, S. Piccarolo, J. Loos, and A. Alexeev (unpublished).

${ }^{7}$ G. Hochstetter, A. Jimenez, and J. L. Loubet, J. Macromol. Sci., Phys. B38, 681 (1999).

${ }^{8}$ V. Brucato, S. Piccarolo, and V. La Carrubba, Chem. Eng. Sci. 57, 4129 (2002).

${ }^{9}$ D. Tranchida and S. Piccarolo, Polymer 46, 4032 (2005).

${ }^{10}$ W. C. Oliver and G. M. Pharr, J. Mater. Res. 19, 3 (2004).

${ }^{11}$ M. Troyon and M. Martin, Appl. Phys. Lett. 83, 863 (2003).

${ }^{12}$ R. B. King, Int. J. Solids Struct. 23, 1657 (1987).

${ }^{13}$ J. C. Hay, A. Bolshakov, and G. M. Pharr, J. Mater. Res. 14, 2296 (1999).

${ }^{14}$ A. Pawlak and A. Galeski, Macromolecules 38, 9688 (2005).

${ }^{15}$ M. S. Bobji, S. K. Biswas, and J. B. Pethica, Appl. Phys. Lett. 711059 (1997).

${ }^{16}$ D. Tranchida, S. Piccarolo, and M. Soliman, Macromolecules 39, 4547 (2006).

${ }^{17}$ M. Saiu, V. Brucato, S. Piccarolo, and G. Titomanlio, Int. Polym. Process. 7, 267 (1992). 